\title{
Correction to: Science of Cyber Security
}

\author{
Wenlian Lu, Kun Sun, Moti Yung, and Feng Liu
}

\section{Correction to: \\ W. Lu et al. (Eds.): Science of Cyber Security, LNCS 13005, https://doi.org/10.1007/978-3-030-89137-4}

In the original version of this book, the affiliation of Feng Liu was presented incorrectly. This has been corrected to Institute of Information Engineering, Chinese Academy of Sciences, Beijing, China. 